\title{
EXPLICIT EXPRESSIONS FOR A CLASS \\ OF PERMUTATION PROBLEMS
}

\author{
Morton Abramson \\ (received April 19, 1963)
}

The purpose of this paper is to give some explicit formulae for probability problems such as those dealing with maximum runs upon tossing a coin $\mathrm{n}$ times in succession. Although the actual computation involved is quite lengthy, and generating functions can be used for approximations, the lemmas obtained may be of some interest in themselves.

Throughout the paper the symbol $\left(\begin{array}{c}a_{i+1} \\ a_{i}\end{array}\right)$ denotes the binomial coefficient where $a_{i}$ and $a_{i+1}$ are non-negative integers and $\left(\begin{array}{l}a_{i+1} \\ a_{i}\end{array}\right)=0$ if $a_{i}>a_{i+1}$. We denote the product $\left(\begin{array}{l}a_{2} \\ a_{1}\end{array}\right)\left(\begin{array}{l}a_{3} \\ a_{2}\end{array}\right) \cdots\left(\begin{array}{l}a_{m} \\ a_{m-1}\end{array}\right)$ by the symbol $\left(\begin{array}{c}a_{m} \\ \vdots \\ a_{2} \\ a_{1}\end{array}\right)$.

LEMMA 1*. Let $A_{j+1}(n, k)$ be equal to the number of ways of choosing $k$ elements from $n$ elements $x_{1}, \ldots, x_{n}$ so that no $j+1$ consecutive elements appear in any choice. Then

This is a generalization of Problem E-1479, Elementary Problems and solutions section of The American Mathematical Monthly, Vol. 69, 1962, ก. 235.

Canad. Math. Bull. vol. 7, no. 3, July 1964 


$$
A_{j+1}(n, k)=\sum_{i_{1}+i_{2}} \sum_{j+i_{j}=k}\left(\begin{array}{c}
n-k+1 \\
i_{j} \\
\vdots \\
i_{2} \\
i_{1}
\end{array}\right), i_{j} \geq i_{j-1} \geq \ldots \geq i_{1} .
$$

Proof. We wish to find the number of distinguishable arrays of $n-k$ dashes and $k$ dots along a straight line with the restriction of $j+1$ consecutive dots appearing. To do this we array only the $n-k$ dashes along a straight line and consider the $n-k+1$ spaces or slots, those between the dashes, the space before the first dash and the space after the last dash. We divide the $k$ dots into $i_{1}$ groups of $j$ consecutive dots each, $i_{2}-i_{1}$ groups of $j-1$ consecutive dots each, $i_{3}-i_{2}$ groups of $j-2$ consecutive dots each, and so on in a like manner until we have $i_{j}-i_{j-1}$ groups of 1 dot each. Also $i_{u} \geq i_{v}$ if $u \geq v$ and necessarily $i_{1}+i_{2}+\ldots+i_{j}=k$. For a fixed selection of such i's there are $\left(\begin{array}{c}i_{j} \\ \vdots \\ i_{2} \\ i_{1}\end{array}\right)$ distinguishable divisions of the $k$ dots into such groups. Thus there are $\left(\begin{array}{c}n-k+1 \\ i_{j} \\ \vdots \\ i_{2} \\ i_{1}\end{array}\right)$ possible distinguishable insertions of the groups of

the division into the $n-k+1$ slots, with at most one group being inserted into any one slot. The lemma follows.

The case when $j=1$ is quite interesting and was first given by Kaplansky [2] as a Lemma, in obtaining the "quickest" 
solution to the "Problème des Ménages".

The number of ways of chocsing $k$ from $n$. so that the maximum number of consecutive elements in each choice is exactly $j$ is denoted by $\bar{A}_{j}(n, k)$; clearly

$$
\bar{A}_{j}(n, k)=A_{j+1}(n, k) \cdot A_{j}(n, k) .
$$

For example, if $p$ is the probability of obtaining a head in a single toss, then

$$
\sum_{k=0}^{n} \sum_{j=k}^{n} \bar{A}_{j}(n, k) p^{k}(1-p)^{n-k}=1 \text {, }
$$

and we have here a probability distribution with the value of the random variable being equal to the length of the maximum 5 un.

$$
\sum_{k=0}^{n} A_{j+1}(n, k) p^{k}(1-p)^{n-k}
$$

is the probability that heads will not appear $j+1$ times in succession.

Further, we denote by $A_{j+1}(n)$ all possible distinguishable choices from among $x_{1}, x_{2}, \ldots, x_{n}$ such that no $j+1$ consecutive elements appear in any choice. That is,

$$
A_{j+1}(n)=\sum_{k=0}^{n} A_{j+1}(n, k) .
$$

\section{LEMMA 2.}

$$
\begin{aligned}
A_{j+1}(n) & =A_{j+1}(n-1)+A_{j+1}(n-2)+\ldots+A_{j+1}(n-j-1) \\
& =\sum_{x=1}^{i+1} A_{j+1}(n-x) .
\end{aligned}
$$


Proof. In order to obtain $A_{j+1}(n)$ we may proceed as follows.

First we find the number of all possible distinguishable choices from among $x_{2}, x_{3}, \ldots, x_{n}$ with the " $j+1 "$ restriction. This is equal to $A_{j+1}(n-1)$. Now each possible choice from among $x_{3}, x_{4}, \ldots, x_{n}$ with the $" j+1 "$ restriction when added to $x_{1}$ gives rise to a choice from among $x_{1}, x_{3}, \ldots, x_{n}$ each containing $x_{1}$ and with the " $j+1 "$ restriction. In fact all such choices arise in this manner and hence we find their total number to be $A_{j+1}(n-2)$. To find all choices each containing both $x_{1}, x_{2}$ but not $x_{3}$, we consider $x_{4}, x_{5}, \ldots, x_{n}$ and find their total number to be $A_{j+1}(n-3)$. We continue in a like manner and stop when we have found the number of possible choices with the " $j+1$ " restriction, each containing $x_{1}, x_{2}, \ldots, x_{j}$, and which is equal to $A_{j+1}(n-j-1)$. By addition the lemma follows.

If we let $p_{n}$ denote the probability that a run of $j+1$ heads is not observed if a true coin is tossed $n$ times, then, as is easily verified,

$$
P_{n}=\frac{1}{2^{n}} A_{j+1}(n)
$$

and

$$
\begin{aligned}
P_{n} & =\frac{1}{2} p_{n-1}+\frac{1}{2^{2}} p_{n-2}+\ldots+\frac{1}{2^{j+1}} p_{n-j-1} \\
& =\sum_{x=1}^{j+1} \frac{p_{n-x}}{2^{x}} \text { where } p_{0}=p_{1}=\ldots=p_{j}=1 .
\end{aligned}
$$

For $j=2$, it can be shown that, $p_{n} \sim \frac{1.236840}{(1.0873778)^{n+1}}$, [Feller, 1]. 
We now consider the probability of neither of the sequences HTH and HHH appearing in $n$ tosses. In the following Lemma 3 , an $H$ is identified : vith an element which is chosen and a $T$ with an element not chosen. Hence in the proof of the Lemma, an $H$ is identified with a dash and $T$ with a dot.

LEMMA 3. The number of different ways of choosing $k$ elements from among $x_{1}, \ldots, x_{n}$ so that no two elements $x_{i}, x_{i+2}(i=1,2, \ldots, n-2)$ a ppear in any choice is equal to

$$
\sum_{i=0}^{\left[\frac{k}{2}\right]}\left(\begin{array}{c}
n-2 k+2+i \\
k-i \\
i
\end{array}\right) .
$$

Proof. We proceed here in the same manner as we would for $A_{3}(n, k)$, that is for $j=2$, in the proof used for Lemma 1 with the additional restriction that no two consecutive slots both contain dots. Hence, in inserting a division of the $k$ dots consisting of $i$ groups of two dots each and $k-2 i$ groups of one dot each into the $n-k+1$ slots we use the formula $A_{2}(n-k+1, k-i)$. The Lemma follows.

Further, these same considerations lead to an explicit expression for the related probability in a permutation problem treated for the first time in the literature in 1956 by Mendelsohn [3]. Here the restrictions imposed on a random permutation of $n$ integers $1,2, \ldots, n$ are: "1 is 2 d", " $n$ is $(n-1)$ th" and for $i=2,3,4, \ldots, n-1, " i$ is $(i-1) t^{\prime \prime}$ and "i is $(i+1) t^{\prime}$ ". Let $(i, j)$ denote the event that. " $i$ is in the $j^{\text {th }}$ position" under the permutation, $i$ and $j$ being integers. Then the problem is to find the probability, denoted by $P_{n}$, that none of the $2(n-1)$ events

$$
(1,2),(2,1),(3,2),(2,3),(3,4),(4,3), \ldots, L
$$

(where $L$ is the event $(n, n-1)$ or $(n-1, n)$ depending upon whether $n$ is even or odd), occur. Here the probability of 
any $k$ events occurring jointly is equal to either $\frac{(n-k) !}{n !}$ or 0 . The probability of the joint occurrence of any two distinct events $(i, j),(1, m)$ is zero only when, $i=1$ or $j=m$. Hence, using the well known, "principle of inclusion and exclusion", together with Lemma 3 , wherein $2(n-1)$ replaces $n$, we have

$$
P_{n}=\sum_{k=0}^{n} \sum_{i=0}^{\left[\frac{k}{2}\right]}\left(\begin{array}{c}
2 n-2 k+i \\
k-i \\
i
\end{array}\right)(-1)^{k} \frac{(n-k) !}{n !}
$$

\section{REFERENCES}

1. W. Feller, An Introduction to Probability Theory and Its Applications, Vol. 2, 2nd Edition, John Wiley, N.Y., Chapt. XI, p. 260.

2. I. Kaplansky, Solution of the "Problème des Ménages", Bull. Amer. Math. Soc., Vol. 49 (1943), pp. 784-785.

3. N.S. Mendelsohn, The Asymptotic Series for a Certain Class of Permutation Problems, Canadian Journal of Math., Vol. 8 (1956), p. 238.

McGill University 